\title{
Apnea central del sueño con patrón de respiración Cheynes-Stokes
}

\author{
Central sleep apnea with Cheyne-Stokes \\ respiration
}

GermÁn Díaz SANTOS, MD.(1); Selene GueRrero, MD. ${ }^{(2)}$

\section{Resumen}

El síndrome de apnea central del sueño con patrón de Cheyne-Stokes es una patología prevalente en los pacientes con falla cardiaca. Ésta influencia la morbilidad y la mortalidad de la enfermedad, por lo cual se realizó una revisión narrativa de la literatura, enfocándonos en la epidemiología, fisiología y tratamiento de la apnea central del sueño con patrón de Cheyne-Stokes.

Palabras clave: apnea central del sueño, respiración de Cheyne-Stokes, presión positiva continua en la vía aérea, servoventilación adaptativa.

\begin{abstract}
Central sleep apnea syndrome with Cheyne-Stokes respiration is a common condition in patients with heart failure. This influences the morbidity and mortality of the disease. For this reason, we carried out a narrative review of the literature, focusing on the epidemiology, physiology, and treatment of central sleep apnea with Cheyne-Stokes respiration.
\end{abstract}

Keywords: central sleep apnea, Cheyne-Stokes respiration, constant positive airway pressure, adaptive servo-ventilation.

\section{Introducción}

La apnea central del sueño es un grupo heterogéneo de trastornos respiratorios relacionados con el sueño, en el que se disminuye el esfuerzo respiratorio o se encuentra ausente de forma intermitente o cíclica durante éste, con diferentes fisiopatologías que generalmente incluyen la concentración de dióxido de carbono. (1). Se clasifica de acuerdo con su causa como apnea central primaria del sueño y apnea central secundaria del sueño. Otra forma de clasificarla es por su fisiopatología, si se presenta con hipercapnia o hipocapnia.

Una apnea central se define mediante polisomnografía como un cese del flujo de aire del noventa por ciento, durante 10 segundos o más, asociado a un esfuerzo respiratorio ineficaz. Una hipopnea central es la reducción en la amplitud de la onda de flujo en el treinta por ciento o más (alternativamente $50 \%$ ) que se acompaña con una disminución de la saturación de más del 3\% (alternativamente $4 \%$ ) o un alertamiento (2).
(1)Epidemiólogo, Internista, Neumólogo, Somnólogo. Somnólogo y Director Médico, Clínica Somnomédica. Bogotá, Colombia. (2)Epidemióloga, Neumóloga, Fisióloga pulmonar. Instituto Nacional de Enfermedades Respiratorias. Ciudad de México, México. Correspondencia: Germán Díaz Santos, correo electrónico: docger25@hotmail.com Recibido: 04/06/17. Aceptado: 06/07/17. 
Cheyne-Stokes es un patrón respiratorio patológico caracterizado por hiperventilación en patrón creciendo-decreciendo con cambios en el volumen corriente, alternado con apneas o hipopneas centrales. Se diagnostica cuando hay tres ciclos respiratorios consecutivos con este patrón y un índice de apnea-hipopnea superior a 5 eventos por hora de sueño, con un periodo mínimo de dos horas. Este se puede presentar durante la noche o en el día, o bajo reposo o ejercicio (3).

Por ser una enfermedad con alta prevalencia sobre todo en pacientes con falla cardiaca y su presencia influenciar de forma importante la sobrevida de los pacientes, el objetivo principal de este trabajo fue realizar una revisión narrativa de la literatura, enfocada en la epidemiología, la fisiología y el tratamiento de la apnea central del sueño con patrón de Cheyne-Stokes.

\section{Epidemiología}

La apnea central del sueño es una entidad con baja incidencia (menor del $1 \%$ en población general y $10 \%$ en pacientes con trastornos respiratorios del sueño) (4).

La presencia de apneas centrales en falla cardiaca es del 25 al 40\%, en enfermedad cerebrovascular del $20 \%$ y con uso de opioides (metadona) del 30\% (5).

La asociación de diferentes patologías con el patrón de Cheyne-Stokes es variable, dependiendo del estudio y los criterios para diagnosticarlo. Sin embargo, hasta el momento por su asociación con pacientes en falla cardiaca (59\%), se considera que estos mismos factores influencian el riesgo para presentar este patrón respiratorio (edad mayor de 60 años, género masculino, $\mathrm{CO}_{2}$ menor de $38 \mathrm{~mm} \mathrm{Hg}$ despierto, fibrilación auricular y fracción de eyección disminuida) (6).

El patrón de Cheyne-Stokes se reporta principalmente durante la noche (62\%), comparada con el día $(16 \%)$ en pacientes con índice de apnea-hipopnea mayor o igual a 15 eventos/hora (7).

Los pacientes con falla cardíaca asociada a apnea central del sueño con patrón de Cheyne-Stokes tienen una tasa de mortalidad dos veces más alta que los que no poseen esta asociación (8). Además, el tener este patrón respiratorio durante el día aumenta la mortalidad en un $10 \%(9)$.

\section{Fisiopatología}

Durante la vigilia la respiración es controlada principalmente por la corteza cerebral, pero durante el sueño, este control se pierde y los estímulos químicos pasan a controlar la ventilación (principalmente $\mathrm{CO}_{2}$ ). En el estado de sueño se presenta normalmente un leve aumento del $\mathrm{CO}_{2}$, con un incremento correspondiente del umbral de apnea, permitiendo que exista un nivel mínimo de $\mathrm{CO}_{2}$ para activar la inspiración. Cuando se presenta una reducción del $\mathrm{CO}_{2}$ por debajo de este umbral, se dan apneas centrales. Esto ocurre normalmente en los periodos de transición de sueño a vigilia $(92 \%$ de los eventos ocurren en fase de sueño N) (10), pero es rápidamente adaptado por el sistema. No obstante, la alteración en los quimiorreceptores e inestabilidad ventilatoria (ganancia de asa) puede llevar a síndrome de apnea central del sueño (11) (figura 1).

\section{Ganancia de asa}

El sistema de asa de realimentación respiratoria, comprende quimiorreceptores periféricos y centrales, receptores vagales intrapulmonares, el centro de control respiratorio en el tronco encefálico y los músculos respiratorios. Lo que se determina como ganancia de asa, representa la estabilidad del asa de realimentación respiratoria de forma cuantitativa. Esta ganancia de asa está regulada por la ganancia de controlador y la ganancia de planta. La ganancia de controlador, representa el grado de respuesta a un disturbio dado (quimiosensibilidad), y la ganancia de planta representa la eficiencia de la respuesta al disturbio (eficacia para eliminar el $\mathrm{CO}_{2}$ durante la ventilación). Esta nos da la magnitud de la respuesta ventilatoria proporcionada por el sistema de control respiratorio a una alteración respiratoria (disturbio respiratorio) (12).

El disturbio respiratorio es un periodo de hiperventilación (reducción del $\mathrm{CO}_{2}$ ), que es detectado por quimiorreceptores, y posterior a un breve 

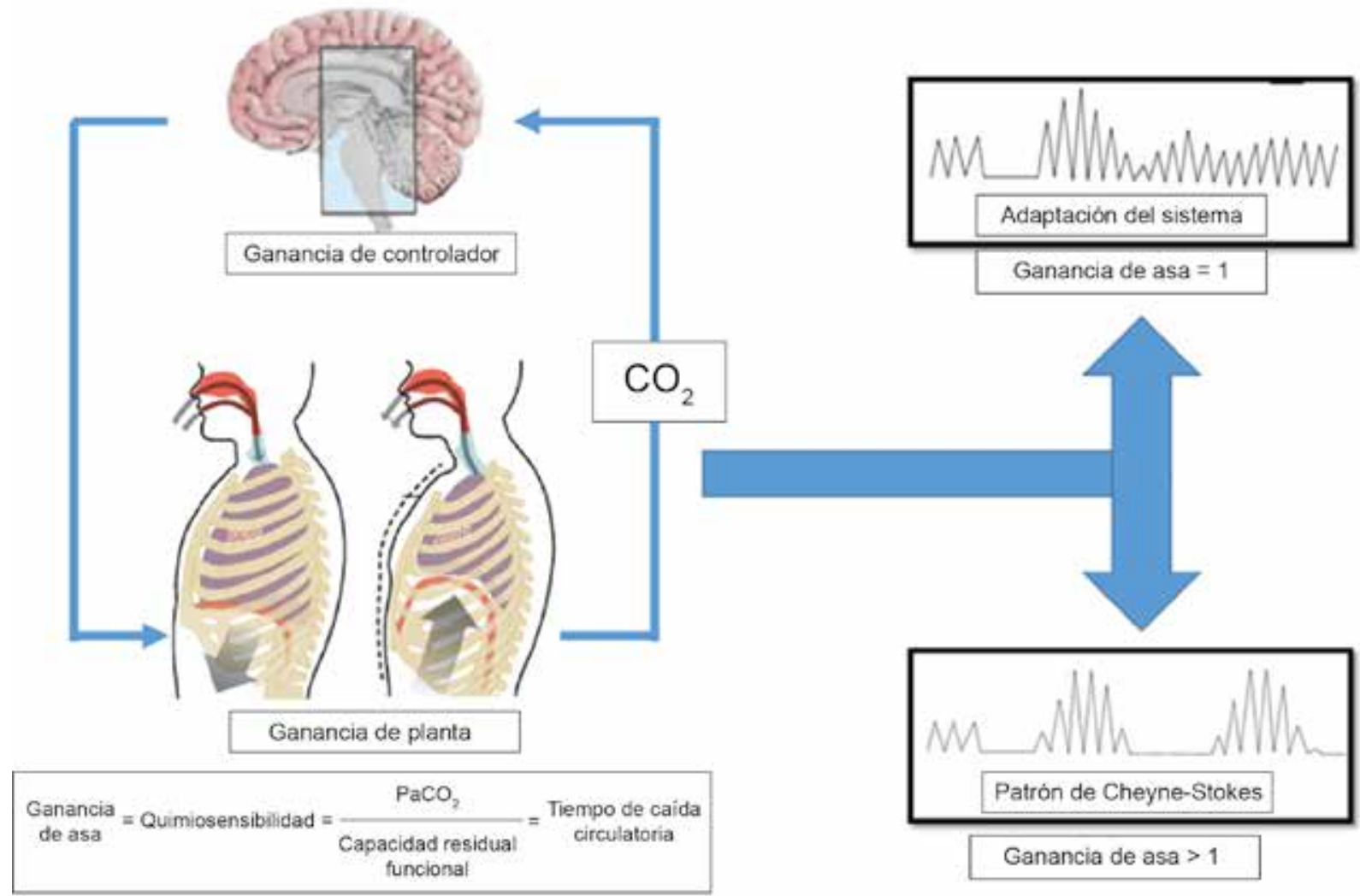

Figura 1. Mecanismos involucrados en la teoría de ganancia de asa para la apnea central del sueño.

periodo de tiempo (denominado caída circulatoria), presenta una respuesta por parte del sistema de control ventilatorio (13) y la resistencia a su supresión con el tratamiento (14).

Cuando la respuesta es mayor que la perturbación, el asa de realimentación, toma un valor de ganancia de asa superior a 1.0, causándose inestabilidad y oscilaciones periódicas en la respiración. Cuando la respuesta es menor que el disturbio, la ganancia de asa es representada como menor que 1 , lo que causa que las oscilaciones transitorias sean atenuadas (15).

\section{Falla cardiaca y apnea central con patrón de respiración de Cheyne-Stokes}

Muchos elementos contribuyen potencialmente a la hiperventilación. Los pacientes con una baja tasa metabólica, espacio muerto disminuido, capacidad residual funcional baja o un alto $\mathrm{PaCO}_{2}$, tienen predisposición a una mayor ganancia de planta por tener cambios ventilatorios marcados. Varios factores pueden contribuir a hiperventilación en estos pacientes, pero los más importantes son la presencia de congestión pulmonar y la quimiosensibilidad exagerada, que hacen que los pacientes con falla cardiaca sean altamente predispuestos a sufrir estos fenómenos. Los factores mejor comprendidos al momento son la congestión pulmonar y la quimiosensibilidad exagerada. Entre otros factores que pueden contribuir al desarrollo del patrón de Cheyne-Stokes están el volumen de la aurícula izquierda, la reactividad vascular cerebral, la hipoxia, la resistencia de la vía aérea superior, la capacidad residual funcional, el retardo circulatorio y la deficiencia de leptina.

La congestión pulmonar lleva a un aumento de la presión de llenado del ventrículo izquierdo y a mayor presión de cuña capilar pulmonar, que empeora con los cambios de posición por aumento de 
fluidos a la zona del cuello, tórax y pulmón provenientes de los miembros inferiores. Parece que también hay una estimulación de fibras del nervio vago sin conocerse del todo su fisiopatología. (16). En pacientes con falla cardiaca se presenta una alta quimiosensibilidad que lleva a fluctuaciones de $\mathrm{CO}_{2}$ y respuesta exageradas a la ventilación, llevando a un círculo vicioso (17).

La respuesta de los quimiorreceptores centrales a los cambios en el $\mathrm{CO}_{2}$ está influida por la reactivad vascular cerebral, ya que no se presenta una respuesta inmediata a la hiperventilación ni a la hipoventilación, por lo cual facilita la aparición de apneas centrales (18). La hiperventilación puede ser potenciada por la hipoxia ya que puede influenciar la respuesta ventilatoria (12). Un aumento del índice de volumen auricular izquierdo $\left(\geq 44 \mathrm{ml} / \mathrm{m}^{2}\right)$ se correlaciona con una quimiosensibilidad aumentada en pacientes con falla cardiaca (19).

Los cambios que se presentan a nivel de la resistencia de la vía aérea superior durante las fases de aumento y disminución de la ventilación en la respiración de Cheyne-Stokes, pueden predisponer a perpetuar también este fenómeno (17). No está muy claro el papel de la reducción de la capacidad residual funcional, ya que estudios muestran que no hay cambios en el volumen pulmonar durante estas patologías; sin embargo estas podrían condicionar mayor retención de $\mathrm{CO}_{2}$ e hipoxemia (20).

Los pacientes con falla cardiaca presentan un retraso en el tiempo de estimulación en los quimiorreceptores (tiempo de caída circulatoria) como consecuencia a un gasto cardiaco disminuido, lo cual predispone a la asociación con respiración de Cheyne-Stokes (21).

Es contradictorio el compromiso de fracción de eyección del ventrículo izquierdo como riesgo para el desarrollo de la respiración de Cheyne-Stokes, ya que en algunos estudios se encontró asociado con baja fracción de eyección y en otros no se se halló correlación $(7,22)$.

Se ha encontrado deficiencia de leptina en un pequeño grupo de pacientes con apnea central del sueño y se correlacionó con un alto nivel de péptido natriurético e hiperventilación durante el reposo y el ejercicio (23).

\section{Tratamiento}

Se debe optimizar la terapia médica de los pacientes con falla cardiaca, ya que este podría ser el desencadenante de los eventos respiratorio. Se ha informado la presencia de apneas centrales hasta en el $75 \%$ de los pacientes con falla cardiaca descompensada (24).

\section{Medicamentos}

Sólo unos pocos estudios no aleatorizados han investigado los efectos de los fármacos cardíacos en pacientes con insuficiencia cardíaca con trastornos respiratorios del sueño.

Desde el punto de vista del manejo de la falla cardiaca, el uso de betabloqueadores mejoró significativamente la función ventricular izquierda, redujo el nivel de péptido natriurético cerebral y el número de apneas centrales (25). Usar inhibidores de la enzima convertidora de angiotensina mostró una mejoría significativa de los trastornos respiratorios del sueño y la calidad subjetiva del sueño en pacientes con insuficiencia cardíaca (26). También se ha estudiado la utilidad de los diuréticos (27).

Otro grupo de fármacos utilizados son los estimuladores respiratorios como acetazolamida, teofilina, dióxido de carbono exhalado, benzodiacepinas, entre otros.

La acetazolamida es un diurético que estimula el control respiratorio y ha demostrado un leve aumento en la ventilación, disminuyendo el índice de apnea hipopnea central sin normalizar los valores de este (28).

Se ha utilizado teofilina en cortos periodos sin mostrar mejoría de los parámetros respiratorios, pero otros estudios muestran mejoría en los niveles de $\mathrm{CO}_{2}$ transcutáneo (29).

Estudios que utilizaron la aplicación de $\mathrm{CO}_{2}$ exhalado, mostraron un aumento del espacio muerto, con normalización de la ventilación en la apnea central del sueño con patrón de Cheyne-Stokes (30). 
El $\mathrm{CO}_{2}$ transcutáneo aumentó y el tiempo con el patrón de respiración de Cheyne-Stokes se redujo significativamente al aplicar una mezcla de oxígeno y dióxido de carbono en pacientes con insuficiencia cardíaca, con elevación secundaria de la activación simpática (31).

Se ha estudiado la aplicación $\mathrm{CO}_{2}$ en diferentes dosis y tiempos del ciclo respiratorio, de forma matemática, encontrando que durante un corto período de tiempo se estabiliza la respiración con un mínimo aumento del $\mathrm{PCO}_{2}$. Sin embargo, no se han realizado estudios clínicos a largo plazo porque una sobredosis de $\mathrm{CO}_{2}$ puede ser potencialmente mortal (32).

También se ha probado con ansiolíticos. En estudios en animales y en ensayos clínicos se ha encontrado que los despertares desestabilizan la ventilación, pudiendo agravar trastornos respiratorios en pacientes con Cheyne-Stokes. Los barbitúricos también pueden disminuir los despertares y estabilizar la ventilación; por lo cual se ha utilizado pentotal, encontrando estabilización de la respiración, con episodios de hipoxemia prolongada (33).

\section{Oxígeno}

Teniendo en cuenta la teoría de la ganancia de asa, suministrar oxígeno puede interferir con este ciclo y reducir la hiperreactividad del sistema al $\mathrm{CO}_{2}$, ya que al llegar mayor cantidad de oxígeno al ventrículo izquierdo, se reduce la activación refleja de los quimiorreceptores periféricos. Algunos trabajos muestran que el suministro de oxígeno normalizó el índice de desaturación y la función ventricular izquierda mejorada. Sin embargo, la reducción de la IAH se limita al 50\% (34); por lo tanto, no hay un consenso para el uso de este ya que puede aumentar la frecuencia de apneas obstructivas en pacientes con enfermedad mixta (35). Los resultados de los ensayos clínicos sobre la eficacia son contradictorios.

\section{Dispositivos de presión positiva}

La presión positiva continua en la vía aérea se considera el estándar de tratamiento en los pacientes con apnea central y respiración de Cheyne-Stokes, ya que reduce el esfuerzo y trabajo respiratorio, disminuye el consumo de oxígeno, la precarga, la presión transmural del ventrículo izquierdo y la demanda de oxígeno al corazón. Existen diferentes estudios para comparar los diferentes dispositivos de presión positiva utilizados al momento, como lo son: CPAP, binivel y servoventilación adaptativa (ASV).

\section{Dispositivo tipo presión positiva continua en la vía aérea}

Los dispositivos de presión positiva continua en la vía aérea (CPAP) y la ventilación con dos niveles de presión positiva en la vía aérea (binivel), pueden mejorar la ventilación/perfusión, reducir la diferencia alvéolo-arterial de oxígeno y aumentar el suministro de oxígeno al corazón.

En estudios comparativos, utilizar CPAP en pacientes con apnea central del sueño con patrón de respiración de Cheyne-Stokes, demostró una mejoría de las diferentes variables alteradas, pero en individuos sanos no se encontraron cambios significativos y el índice cardiaco disminuyó con el uso del dispositivo. (36).

Algunos estudios muestran que el dispositivo tipo CPAP suprime los trastornos respiratorios del dormir haciendo innecesario el tratamiento con otros dispositivos. Reduce el número de eventos por hora en el $50 \%$ a corto y largo plazo (37) .

Puede mejorar la sobrevida y aumentar la fracción de eyección del ventrículo izquierdo (38).

Otros estudios no muestran superioridad del uso de CPAP en comparación con el tratamiento médico en términos de sobrevida, y análisis posteriores concluyen que el subgrupo de pacientes con respiración de Cheyne-Stokes presenta una mejoría en la sobrevida $(37,39)$.

La terapia de presión positiva de dos niveles se usa en los pacientes con apnea central del sueño con patrón de Cheyne-Stokes y que no responden a la CPAP. Sin embargo, estudios con limitaciones metodológicas, muestran mejoría en las alteraciones respiratorias, parámetros de calidad en el sueño y aumento de la función ventricular izquierda, lo cual 
hace difícil recomendar el uso de estos dispositivos en pacientes con estas características. Existe escaza literatura sobre el uso de CPAP vs. Binivel, encontrando múltiples similitudes entre estos dispositivos, pero solo existe la recomendación de cambiar inmediatamente a ASV si la CPAP no es insuficiente para eliminar los eventos (40).

\section{Servoventilación adaptativa}

Teniendo en cuenta la fisiopatología y la presentación clínica de la apnea central con patrón de Cheyne Stokes se creó la servoventilación adaptativa (ASV) la cual compensa el cambio entre hipernea e hipoventilación mediante la aplicación de una presión de soporte variable. El término servoventilación engloba tres algoritmos comerciales diferentes (41) (tabla 1).

Estudios han comparado las diferentes terapias (oxígeno, CPAP, binivel y servoventilación adaptativa) y ha hallado normalización del índice de apnea central residual comparado con las otras terapias, a corto plazo (42).

Con un mes, el uso de servoventilación adaptativa en pacientes con apnea central con patrón con Cheyne-Stokes, mostró mejoría en marcadores cardiovasculares y la respuesta simpática (43).

El uso de CPAP puede mejorar alteraciones respiratorias, función cardiaca, calidad de vida y apego en comparación con la servoventilación adaptativa; sin embargo, no mostró mejoría en la somnolencia diurna en pacientes con falla cardiaca crónica y apnea central con patrón de Cheyne-Stokes (44).

Se ha comparado binivel con servoventilación adaptativa y se ha visto superioridad en el uso de esta última (45).

Un pequeño grupo de pacientes con falla cardíaca crónica después de haber sido tratadas con CPAP o binivel durante más de 6 meses con disminución del $50 \%$ de los eventos respiratorios, se normalizó al ser tratado con servoventilación adaptativa (46).

En pacientes sin falla cardiaca y apnea central con patrón de Cheyne-Stokes y apnea obstructiva, la servoventilación adaptativa mejoró los trastornos respiratorios del dormir y la calidad del sueño en dos estudios con pocos pacientes, corto seguimiento y sin cambios desde el punto de vista cardiovascular. (41).

Otro estudio en pacientes con falla cardiaca con apnea central con patrón de Cheyne-Stokes y apnea obstructiva en el que se comparó CPAP vs. servoventilación adaptativa, mostró mejoría de las alteraciones respiratorias durante el sueño con servoventilación adaptativa a largo plazo (47).

Los principales eventos adversos que se presentan con el uso de la servoventilación adaptativa fueron la presencia de fugas por la interfaz y la aparición de

Tabla 2. Descripción de los algoritmos que usan los dispositivos de servoventilación adaptativa.

\begin{tabular}{|ll|}
\hline \multicolumn{1}{|c|}{ Algoritmo } & \multicolumn{1}{c|}{ Características } \\
Servoventilación adaptativa & $\begin{array}{l}\text { Durante la hipoventilación, la diferencia entre la presión inspiratoria y espiratoria, } \\
\text { crea un volumen corriente, el cual aumenta en la apnea y se reduce durante la } \\
\text { hiperventilación. }\end{array}$ \\
& $\begin{array}{l}\text { La presión positiva espiratoria es la encargada de eliminar las apneas e hipopneas } \\
\text { obstructivas. }\end{array}$ \\
\hline Auto servoventilación & Para el manejo de las apneas centrales se usas respiraciones de respaldo. \\
Ventilación modulada anticíclica & Frecuencia respiratoria de respaldo y niveles de presión máxima y mínimas variables. \\
& $\begin{array}{l}\text { Combina servoventilación adaptativa con auto-CPAP y alivio de presión, lo cual hace } \\
\text { que varíe la presión soporte y la presión espiratoria para superar la obstrucción de la } \\
\text { vía aérea superior. }\end{array}$ \\
\hline
\end{tabular}


apnea emergente en el tratamiento dada la presencia de presiones muy elevadas (48).

Los últimos metaanálisis sobre el uso de servoventilación adaptativa, muestran aumento de la mortalidad en pacientes con falla cardiaca con fracción de eyección menor del $45 \%$ asociado a apnea central moderada o grave, por lo cual no se recomienda el uso de estos dispositivos en este grupo de pacientes (49).

\section{Quirúrgico}

En los últimos años han aparecido una serie de opciones terapéuticas de tipo quirúrgico para los pacientes con falla cardiaca y patrón de respiración de Cheyne-Stokes, los cuales se describen brevemente a continuación.

\section{Estimulación del nervio frénico}

La estimulación transvenosa del nervio frénico unilateral, reduce los índices de apnea central y mejora el patrón respiratorio en pacientes con falla cardíaca, con base en una pequeña muestra y corto seguimiento (50).

\section{Terapia de resincronización cardiaca}

Un reporte de caso con un dispositivo biventricular seguido de trasplante de corazón indicó que se puede mejorar la disfunción sistólica ventricular izquierda y las apneas centrales, pero solo optimizar de manera transitoria las apneas obstructivas (51).

\section{Trasplante cardiaco}

El efecto de la restauración de la función del corazón a la normalidad con el trasplante de corazón en pacientes con falla cardiaca con respiración de Cheyne-Stokes sólo se ha informado a las pocas semanas del trasplante y con resultados diversos. Algunos persisten con eventos centrales y cuando existen con eventos obstructivos estos reaparecen con el tiempo (52).

\section{Conclusión}

La apnea central del sueño con respiración de Cheyne-Stokes, tiene morbi-mortalidad muy importante en pacientes con falla cardiaca. Se describen múltiples teorías que podrían explicar esta asociación, aunque no todas ellas están totalmente comprobadas. Su manejo debe ser individualizado y elegirse según las opciones terapéuticas. Se requieren más estudios para poder conocer la fisiopatología y posibles esquemas de tratamiento de estos pacientes.

\section{Bibliografía}

1. Panossian LA, Avidan AY. Review of sleep disorders. Med Clin North Am. 2009;93(2):407-25.

2. The AASM Manual for the Scoring of Sleep and Associated Events. Summary of Updates in Version 2.2. July 1, 2015.

3. The International Classification of Sleep Disorders, third edition (ICSD3), 1 published in 2014.

4. Bixler EO, Vgontzas AN, Ten Have T, Tyson K, Kales A. Effects of age on sleep apnea in men: I. Prevalence and severity. Am J Respir Crit Care Med. 1998;157(1):144-8.

5. Wang D, Teichtahl H, Drummer O, et al. Central sleep apnea in stable methadone maintenance treatment patients. Chest. 2005;128(3):1348-56.

6. Johansson P, Alehagen U, Svanborg E, Dahlstrom U, Brostrom A. Sleep disordered breathing in an elderly community-living population: Relationship to cardiac function, insomnia symptoms and daytime sleepiness. Sleep Med. 2009;10(9):1005-11.

7. Poletti R, Passino C, Giannoni A, Zyw L, et al. Risk factors and prognostic value of daytime Cheyne-Stokes respiration in chronic heart failure patients, Int J Cardiol. 2009;137:47-53.

8. Flinta I, Ponikowski P. Relationship between central sleep apnea and Cheyne-Stokes respiration. Int J Cardiol. 2016;206:S8-S12.

9. Luo Q, Zhang HL, Tao XC, Zhao ZH, Yang YJ, Liu ZH. Impact of untreated sleep apnea on prognosis of patients with congestive heart failure. Int J Cardiol. 2010;29:144(3):420-2.

10. Pinna GD, Robbi E, Pizza F, Caporotondi A, et al. Sleepwake fluctuations and respiratory events during CheyneStokes respiration in patients with heart failure. J Sleep Res. 2014;23:349-59.

11. Becker K, Byrd RP. Central sleep apnea syndromes. Disponible en: http://emedicine.medscape.com/article/304967overview\#showall

12. Yumino D, Bradley TD. Central sleep apnea and CheyneStokes Respiration. Proc Am Thorac Soc. 2008;5:226-36.

13. Francis DP, Willson K, Davies LC, et al. Quantitative general theory for periodic breathing in chronic heart failure and its clinical implications. Circulation. 2000;102(18):2214-21.

14. Sands SA, Edwards BA, Kee K, et al. Loop gain explains the resolution of Cheyne-Stokes respiration using inspired $\mathrm{CO} 2$ in patients with heart failure. Am J Respir Crit Care Med, 2012;185:A6697. 
15. Wellman AS, Jordan A, Malhotra et al. Ventilatory control and airway anatomy in obstructive sleep apnea. Am J Respir Crit Care Med. 2004;170(11):1225-32.

16. Brack T. Cheyne-Stokes respiration in patients with congestive heart failure. Swiss Med Wkly. 2003;133:605-10.

17. Lorenzi-Filho G, Genta PR, Figueiredo AC, and Inoue D. Cheyne-Stokes respiration in patients with congestive heart failure: causes and consequences. Clinics. 2005;60:333-44.

18. Costanzo MR, Khayat R, Ponikowski P, Augostini R, et al. Mechanisms and clinical consequences of untreated central sleep apnea in heart failure, JACC. 2015;65(1):72-84.

19. Calvin AD, Somers VK, Johnson BD, Scott ChG, Olson LJ. Left atrial size, chemosensitivity, and central sleep apnea in heart failure. Chest. 2014;146(1):96-103.

20. Naughton M, Benard D, Tam A, Rutherford R, Bradley TD. Role of hyperventilation in the pathogenesis of central sleep apnea in patient with congestive heart failure. Am Rev Respir Dis. 1993;148(2):330-38.

21. Oldenburg T, Bitter M, Wiemer et al. Pulmonary capillary wedge pressure and pulmonary arterial pressure in heart failure patients with sleep-disordered breathing. Sleep Med. 2009;10(7)726-30.

22. Lanfranchi VK, Somers A, Braghiroli U, Corra et al. Central sleep apnea in left ventricular dysfunction: prevalence and implications for arrhythmic risk, Circulation. 2003;107:72732.

23. Cundrle Jr., Somers VK, Singh P, Johnson BD. Leptin deficiency promotes central sleep apnea in patients with heart failure. Chest. 2014;145(1):72-8.

24. Sands SA, Owens RL. Congestive heart failure and central sleep apnea. Sleep Med Clin. 2016;11(1):127-42. doi: 10.1016/j.jsmc.2015.10.003.

25. Tamura A, Kawano Y, Kadota J. Carvedilol reduces the severity of central sleep apnea in chronic heart failure. Circ J. 2009;73:295-98.

26. Walsh JT, Andrews R, Evans A, Cowley AJ. Failure of "effective" treatment for heart failure to improve normal customary activity. Br Heart J. 1995;74:373-76.

27. Solin P, Bergin P, Richardson M, Kaye DM, Walters EH, Naughton MT. Influence of pulmonary capillary wedge pressure on central apnea in heart failure. Circulation. 1999;99(12):1574-9.

28. Javaheri S. Acetazolamide improves central sleep apnea in heart failure: a double-blind, prospective study. Am J Respir Crit Care Med. 2006;173:234-7.

29. Andreas S, Reiter H, Luthje L, Delekat A, Grunewald RW, Hasenfuss G, et al. Differential effects of theophylline on sympathetic excitation, hemodynamics, and breathing in congestive heart failure. Circulation. 2004;110:2157-62.

30. Lorenzi-Filho G, Rankin F, Bies I, Douglas BT. Effects of inhaled carbon dioxide and oxygen on Cheyne-Stokes res- piration in patients with heart failure. Am J Respir Crit Care Med. 1999;159:1490-8.

31. Andreas S, Weidel K, Hagenah G, Heindl S. Treatment of Cheyne-Stokes respiration with nasal oxygen and carbon dioxide. Eur Respir J. 1998;12:414-9.

32. Mebrate Y, Willson K, Manisty CH, Baruah R, Mayet J, Hughes AD, et al. Dynamic CO2 therapy in periodic breathing: a modeling study to determine optimal timing and dosage regimes. J Appl Physiol. 2009;107:696-706.

33. Younes M. Role of arousals in the pathogenesis of obstructive sleep apnea. Am J Respir Crit Care Med. 2004;169:623-33.

34. Sasayama S, Izumi T, Seino Y, Ueshima K, Asanoi H. Effects of nocturnal oxygen therapy on outcome measures in patients with chronic heart failure and Cheyne-Stokes respiration. Circ J. 2006;70:1-7.

35. Gold AR, Bleecker ER, Smith PL. A shift from central and mixed sleep apnea to obstructive sleep apnea resulting from lowflow oxygen. Am Rev Respir Dis. 1985;132:220-3.

36. Naughton MT, Liu PP, Bernard DC, Goldstein RS, Bradley TD. Treatment of congestive heart failure and Cheyne-Stokes respiration during sleep by continuous positive airway pressure. Am J Resp Crit Care Med. 1995a;151:92-7.

37. Bradley TD, Logan AG, Kimoff RJ, Series F, Morrison D, Ferguson $\mathrm{K}$, et al. Continuous positive airway pressure for central sleep apnea and heart failure. N Engl J Med 2005;353:202533.

38. Sin DD, Logan AG, Fitzgerald FS, Liu PP, Bradley TD. Effects of continuous positive airway pressure on cardiovascular outcomes in heart failure patients with and without Cheyne-Stokes respiration. Circulation. 2000;102:61-6.

39. Arzt M, Floras J, Logan A, et al. Suppression of central sleep apnea by continuous positive airway pressure and transplantfree survival in heart failure: a post hoc analysis of the Canadian continuous positive airway pressure of patients with central sleep apnea and heart failure trial (CANPAP). Circulation. 2007;115:3173-80.

40. Köhnlein T, Welte T, Tan LB, Elliott MW. Assisted ventilation for heart failure patients with CheyneStokes respiration. Eur Resp J. 2002;20:934-41; DOI: 10.1183/09031936.00.02622001.

41. Randerath WJ, Galetke W, Kenter M, Richter K, Schafer T. Combined adaptive servo-ventilation and automatic positive airway pressure (anticyclic modulated ventilation) in co-existing obstructive and central sleep apnea syndrome and periodic breathing. Sleep Med. 2009;10:898-903.

42. Teschler H, Dohring J, Wang Y, Berthon-Jones M. Adaptive pressure support servo-ventilation: a novel treatment for Cheyne-Stokes respiration in heart failure. Am J Respir Crit Care Med. 2001;164:614-9.

43. Pepperell JC, Maskell NA, Jones DR, et al. A randomized controlled trial of adaptive ventilation for Cheyne-Stokes breathing in heart failure. Am J Respir Crit Care Med. 2003;168(9):1109-14. 
44. Philippe C, Stoïca-Herman M, Drouot X, et al. Compliance with and effectiveness of adaptive servoventilation versus continuous positive airway pressure in the treatment of Cheyne-Stokes respiration in heart failure over a six month period. Heart. 2006;92(3):337-42.

45. Morgenthaler TI, Gay PC, Gordon N, Brown LK. Adaptive servoventilation versus noninvasive positive pressure ventilation for central, mixed, and complex sleep apnea syndromes. Sleep. 2007;30(4):468-75.

46. Arzt M, Wensel R, Montalvan S, et al. Effects of dynamic bilevel positive airway pressure support on central sleep apnea in men with heart failure. Chest. 2008;134:61-6.

47. Kasai T, Usui Y, Yoshioka T, Yanagisawa N, Takata Y, Narui $\mathrm{K}$, et al. JASV Investigators. Effect of flow-triggered adaptive servo-ventilation compared with continuous positive airway pressure in patients with chronic heart failure with coexisting obstructive sleep apnea and Cheyne-Stokes respiration. Circ Heart Fail. 2010;3(1):140-8.

48. Pusalavidyasagar SS, Olson EJ, Gay PC, Morgenthaler TI. Treatment of complex sleep apnea syndrome: a retrospective comparative review. Sleep Med. 2006;7(6):474-9.
49. Aurora RN, Bista SR, Casey KR, Chowdhuri S, Kristo DA, Mallea JM, et al. Updated Adaptive Servo-Ventilation Recommendations for the 2012 AASM Guideline: "The Treatment of Central Sleep Apnea Syndromes in Adults: Practice Parameters with an Evidence-Based Literature Review and Meta-Analyses”. J Clin Sleep Med. 2016;12(5):757-61. doi: $10.5664 / j \operatorname{csm} .5812$.

50. Ponikowski P, Javaheri S, Michalkiewicz D, Bart BA, Czar necka D, Jastrzebski M, et al. Transvenous phrenic nerve stimulation for the treatment of central sleep apnoea in heart failure. Eur Heart J. 2012;33(7):889-94. doi: 10.1093/eurheartj/ ehr298. Epub 2011 Aug 19.

51. Vermes E, Fonkoua H, Kirsch M, Damy T, Margarit L, Hillion ML, et al. () Resolution of sleep-disordered breathing with a biventricular assist device and recurrence after heart transplantation. J Clin Sleep Med. 2009;5:248-50.

52. Mansfield DR, Solin P, Roebuck T, Bergin P, Kaye DM, Naughton MT. The effect of successful heart transplant treatment of heart failure on central sleep apnea. Chest. 2003;124(5):167581. 